\title{
Atitudes Frente ao Uso de Álcool, Maconha e Outras Drogas: Verificando Relações de Predição e Mediação
}

\author{
Attitudes Toward Alcohol, Marijuana and Other Drugs Use: Verifying \\ Relations of Prediction and Mediation
}

\author{
Carlos Eduardo Pimentel ${ }^{*}, a$, Leconte de Lisle Coelho Júnior ${ }^{b} \&$ Thaís Araújo Aragão ${ }^{b}$ \\ ${ }^{a}$ Universidade de Brasilia \& ${ }^{b}$ Universidade Federal do Espírito Santo
}

\begin{abstract}
Resumo
O objetivo desta pesquisa consistiu em verificar as relações entre atitudes frente ao uso de álcool, maconha e drogas. Contou-se com uma amostra não-probabilística de 276 universitários, com idade média de 21 anos (DP $=3,40)$, de diversos cursos de universidade pública $(94,2 \%)$ e particular $(5,8 \%)$ da cidade de João Pessoa - PB. Estes estudantes responderam às escalas de atitudes frente ao uso de álcool, maconha e drogas. De acordo com as principais análises, pode-se verificar que as atitudes frente ao uso de álcool predizem as atitudes frente ao uso de maconha e drogas, e as atitudes frente ao uso de maconha mediam e moderam a relação entre atitudes frente ao uso de álcool e drogas, independente do sexo e da idade.

Palavras-chave: Atitudes; álcool; maconha; drogas; mediação.
\end{abstract}

\begin{abstract}
The goal of this research consisted in verifying the relations between attitudes towards the use of alcohol, marijuana and other drugs. The study counted with a non-probabilistic sample of 276 academic students, with average age of 21 years old $(S D=3.40)$, from different courses from public $(94.2 \%)$ and private $(5.8 \%)$ universities in the city of João Pessoa - PB. Those students answered attitude scales toward the use of alcohol, marijuana and drugs. According to the main analysis, it was verified that the attitudes toward drug use predict the attitudes related to marijuana and drug use, and that the attitudes toward marijuana use mediate and moderate the relations between the attitudes related to the use of alcohol and drugs, apart from gender and age. Keywords: Attitudes; alcohol; marijuana; drugs; mediation.
\end{abstract}

As atitudes há muito têm sido empregadas para predizer uma miríade de comportamentos, nem sempre de maneira bem sucedida, se tornando em um tópico de grande controvérsia nas ciências sociais (Ajzen, 2001; Kim \& Hunter, 1993; Wicker, 1969). Porém, variáveis relacionadas, como a acessibilidade e relevância atitudinal (Kim \& Hunter, 1993), normas grupais (Smith \& Terry, 2003), força e objeto das atitudes, podem incrementar esta relação (Holland, Verplanken, \& van Knippenberg, 2002). No caso específico das atitudes frente ao uso de drogas, estas têm sido consistentes na predição do comportamento de uso de drogas (para uma revisão ver Hawkins, Catalano, \& Miller, 1992; Petraits, Flay, \& Miller, 1995; ver também Holland et al., 2002; Simons \& Carey, 1998), assim como as atitudes frente ao uso de álcool predizem o uso de álcool (Petraits et al., 1995; Simons \& Gaher, 2004).

A teoria da ação racional (TAR; Ajzen \& Fishbein, 1980; Fishbein \& Ajzen, 1975) explica que o uso de drogas psicotró-

* Bolsista do CNPq. Endereço de correspondência: Universidade de Brasília, Doutorado em Psicologia Social, do Trabalho e das Organizações. Campus Darcy Ribeiro, Brasília DF, Brasil, CEP 10910-900. E-mail: carlospimentel@unb.br ou carlosepimentel@bol.com.br picas é determinado pelas intenções e atitudes frente ao uso, além das normas sociais. Estas atitudes e crenças sociais normativas sobre o uso de drogas predizem satisfatoriamente seu uso (Ajzen, Timko, \& White, 1982). Portanto, conhecer as atitudes frente às drogas auxiliaria a prática médica, psiquiátrica e psicológica (Isacson \& Bingefors, 2002), além do controle e prevenção do uso (Blaya \& Antón, 1993; Hawkins et al., 1992; Isacson \& Bingefors, 2002; National Institute on Drug Abuse [NIDA], 2003; Petraits et al., 1995; Scott, 1996; Simons \& Carey, 1998; Simons $\&$ Gaher, 2004). De fato, as atitudes desviantes e contra as drogas são tidas em apreço em princípios (baseados em pesquisas) de prevenção do uso de drogas do NIDA (2003). Entre estudantes universitários brasileiros, verificam-se características importantes no consumo de álcool: estes estudantes bebem "se embriagando" (binge), assim como abusam de outras drogas e têm atitudes favoráveis frente a drogas ilícitas (Kerr-Corrêa, Andrade, Bassit, \& Boccuto, 1999). Este padrão de atitudes de usuários de álcool e tabaco tem sido verificado em outro contexto (Best et al., 2000).

Outros estudos têm oferecido suporte empírico à relação entre as atitudes e comportamentos de uso de substâncias lícitas e ilícitas. Simons e Carey (2000) verificaram que as atitudes frente ao uso de maconha predizem significativa e 
estatisticamente $(p<0,001)$ o comportamento de uso (Estudo 1: $\beta=0,53$ e Estudo 2: $\beta=0,41$ ) e as atitudes frente ao não-uso de drogas modera esta relação. EnriquezFlores e Luis (2004) realizaram uma pesquisa sobre atitudes e uso de álcool, tabaco, folhas de coca, tranqüilizantes e anfetaminas e verificaram que as atitudes favoráveis frente às drogas corresponderam aos maiores índices de aprovação do uso de álcool, folhas de coca e tranqüilizantes. Gouveia, Pimentel, Medeiros, Gouveia e Palmeira (2007) observaram o poder preditivo das atitudes frente ao uso de drogas com relação ao comportamento de uso $(B=0,17$; $O R=1,19, p=0,004)$ entre estudantes universitários. Destes participantes, apenas 1,5\% admitiu ser usuário de algum tipo de droga (como cocaína, crack e ecstasy), manifestando, na grande maioria, atitudes muito negativas frente às drogas. Foi verificado ainda que as mulheres apresentaram atitudes mais negativas frente ao uso de drogas em geral. Esta relação foi anteriormente observada (Gouveia, Pimentel, Queiroga, Meira, \& Jesus, 2005) em alunos secundaristas, entre os quais estudantes do sexo feminino apresentaram atitudes mais negativas frente à maconha em comparação aos estudantes do sexo masculino; além disso, os com idades acima de 16 anos foram mais favoráveis do que os com idades abaixo de 16 anos.

Colle e Curtet (1983) afirmam que erroneamente a mídia e a população em geral assumem uma postura de acreditar que as drogas ilegais, como a maconha, são aquelas que iniciam as pessoas numa escalada de consumo. De acordo com a idéia da "escalada de consumo", uma vez consumida uma droga ilegal, ela servirá de degrau para outras mais pesadas, quaisquer que sejam (Colle \& Curtet, 1983). As teorias que tratam do desenvolvimento, ou estágios, no uso de drogas (chamadas pathways theory of drug use) afirmam que o indivíduo inicia o uso das drogas chamadas lícitas, "leves", softs, como álcool e tabaco, passando pela maconha, para progressivamente usar drogas "pesadas", hards, como a cocaína e a heroína (Ellickson, Hays, \& Bell, 1992). Porém, deve-se destacar que é possível que o indivíduo pule o estágio do uso da maconha e passe diretamente para o uso de drogas mais pesadas, ou seja, das lícitas, álcool e tabaco, para as drogas mais fortes e ilícitas (BlazeTemple \& Lo, 1992). Destaque-se ainda que a importância de substâncias, como o álcool e a maconha, que instigam o uso de outras substâncias, tem mudado ao longo do tempo, com maiores taxas de consumo de uma ou outra droga (Golub \& Johnson, 1994). No entanto, alguns pesquisadores (Coelho Jr. \& Gontiès, 2000; Noto et al., 2003) já afirmam que as drogas mais perigosas são aquelas ditas legais, pois estas têm todo um aparato jurídico que protege a sua fabricação e consumo, como é o caso dos medicamentos (Roazzi, 1987). Inclusive, a mídia pode ser entendida como um aparato que estimula o aumento do consumo. Sanchez e Nappo (2002) mostraram em sua pesquisa que bebidas alcoólicas, cigarro e solventes foram as primeiras a serem utilizadas por seus informantes. Por outro lado, do grupo das drogas ilegais, é a maconha que surge como primeira opção.
O conhecimento das inter-relações entre estas atitudes pode ajudar a entender como se desenvolve o uso de drogas pesadas, mais prejudiciais à saúde, como cocaína, LSD, crack e drogas sintéticas. A presente pesquisa foi conduzida a fim de verificar relações (de predição e mediação) entre atitudes frente ao uso de álcool, maconha e outras drogas e, assim, contribuir para a psicologia das atitudes frente ao uso de substâncias. Não foi encontrado nenhum estudo que verificasse associações entre atitudes frente ao álcool, maconha e outras drogas. Objetivou-se observar, especificamente, se as atitudes frente ao álcool predizem atitudes frente à maconha e se estas últimas predizem as atitudes frente às drogas (Atitudes frente ao álcool $\rightarrow$ Atitudes frente à maconha $\rightarrow$ Atitudes frente às drogas). Buscar-se-á ainda conhecer o poder preditivo do sexo e da idade nas atitudes frente às drogas, pois pesquisas demonstram que estas variáveis são influentes no uso, nas atitudes frente ao uso e em outras variáveis relacionadas ao uso (Correia, Simons, Carey, \& Borsari, 1998; Donnermeyer \& Huang, 1991; Gouveia et al., 2007; Gouveia et al., 2005; Hawkins et al., 1992; Petraits et al., 1995; Shedler \& Block, 1990).

\section{Método}

\section{Participantes}

Tratou-se de uma amostra de conveniência (não-probalística) composta por 276 estudantes universitários, provenientes de uma universidade pública $(94,2 \%)$ e outra particular (5,8\%), localizadas na cidade de João Pessoa - PB. Os participantes eram de diversos cursos (Administração, Ciências Sociais, Computação, Direito etc.), sendo que a maioria $(54,7 \%)$ se distribuiu entre os cursos de Ciências Contábeis $(30,8 \%)$ e Psicologia $(23,9 \%)$. A idade destes variou de 17 a 43 anos $(m=21 ; D P=3,40)$, com a maioria formada por jovens de 17 a 25 anos $(93,5 \%)$. Predominaram aqueles do sexo feminino $(65,6 \%)$, solteiros $(90,6 \%)$ e que se consideravam de classe sócio-econômica média $(59,5 \%)$ ou média-baixa $(28,1 \%)$.

\section{Instrumentos}

Foram utilizados nesta pesquisa: Escala de Atitudes Frente ao Uso de Álcool (EAFUA), Escala de Atitudes Frente ao Uso de Maconha (EAFUM) e Escala de Atitudes Frente ao Uso de Drogas (EAFUD). Todas estas medidas são auto-administráveis (self-report) e seguem a forma da EAFUM, adaptada por Gouveia et al. (2005), pautada nas escalas desenvolvidas por Crites, Fabrigar e Petty (1994) para vários objetos atitudinais, sendo utilizadas por Simons e Carey (2000) para o uso de maconha e álcool (ver também Simons \& Gaher, 2004). Cada instrumento usado baseia-se em 4 escalas de diferencial semântico, e consiste em saber a avaliação global do jovem acerca de estar sob o efeito de bebidas alcoólicas (4 itens), estar sob o efeito de maconha (4 itens) e estar sob o efeito de drogas (como cocaína, crack ou ecstasy, 4 itens). Os quatro pares de adjetivos, itens, (positivo/negativo, agradável/desagradável, bom/ruim e desejável/indesejável) são situados nos extre- 
mos do diferencial semântico de 9 pontos. Como na versão da EAFUM, as pontuações 1, 2, 3 e 4 representam atitudes favoráveis, sendo 5 o ponto nulo, enquanto as pontuações 6, 7, 8 e 9 indicam atitudes desfavoráveis frente ao uso. $\mathrm{O}$ participante, portanto, deveria se basear na frase "Considero estar sob o efeito de álcool..." e responder no continuum do diferencial semântico (positivo/negativo, agradável/ desagradável, bom/ruim e desejável/indesejável). Esta frase estímulo também era usada para maconha e drogas.

No estudo de Gouveia et al. (2005) corroborou-se esta estrutura uni fatorial, com variância total explicada de $84,1 \%$ e índice de consistência interna (Alfa de Cronbach) de 0,94 . Realizou-se ainda uma análise fatorial confirmatória, tendo sido observados os seguintes índices de ajuste: $\chi^{2} /$ g.l. $=0,87, G F I=1, A G F I=0,99$ e $R M R=0,01$, reunindo, assim, evidências favoráveis de validade fatorial e precisão, que justificam seu emprego em pesquisas realizadas neste país.

Questionário Demográfico. Constituiu-se por um conjunto de perguntas: idade, sexo, estado civil, classe sócioeconômica percebida, universidade onde estuda e curso que faz, a fim de caracterizar os participantes do estudo, figurando como última parte a ser respondida.

\section{Procedimento}

Os aplicadores dos instrumentos foram os próprios autores do presente artigo, os quais buscaram não intervir nas respostas dos participantes, minimizando a possibilidade de algum viés de resposta. A aplicação ocorreu de forma coletiva nas salas de aula, bastando aos participantes seguirem as orientações dadas por escrito no próprio questionário. Os aplicadores permaneceram em sala, atentos para possíveis dúvidas quanto à forma de responder. Procurou-se ainda oferecer informações oralmente sobre aspectos éticos da pesquisa, o que consistiu em: (a) assegurar a participação voluntária e consentida; (b) o sigilo das respostas, que deveriam ser tratadas estatística e coletivamente; (c) solicitar aos jovens que não se identificassem; (d) que poderiam declinar da participação na pesquisa a qualquer momento e (e) que aquela pesquisa poderia ser útil para a sociedade e o conhecimento científico da psicologia. A aplicação teve uma duração média de quinze minutos. Foram salvaguardados todos os procedimentos éticos para a realização de pesquisas com seres humanos (risco mínimo, direito ao sigilo, consentimento informado), de acordo com a American Psychological Association ([APA], 2002), respeitando a dignidade e o direito das pessoas. Neste sentido, anexo à escala, constava um termo de consentimento livre e esclarecido que deveria ser rubricado pelo participante. Respondidos os instrumentos, estes eram rapidamente checados e, finalmente, expressavam-se os agradecimentos aos estudantes e professores presentes durante a coleta dos dados.

\section{Resultados}

Os resultados foram obtidos mediante Análises de Correlação (de ordem zero e parcial) e Análises de Regressão (linear simples e múltipla). Foram verificadas inicialmente correlações entre as três variáveis em análise, atitudes frente ao álcool, maconha e drogas (Tabela 1). De acordo com a análise, puderam-se verificar associações estatisticamente significativas entre as atitudes (todas com $p<$ 0,01).

Tabela 1

Correlações entre Atitudes frente ao Álcool, Maconha e Drogas

\begin{tabular}{llllll}
\hline & M & DP & AFA & AFM & AFD \\
\hline AFA & 6,53 & 2,39 & 1 & & \\
AFM & 7,44 & 2,06 & $0,60 * *$ & 1 & \\
AFD & 7,97 & 1,71 & $0,56 * *$ & $0,76 * *$ & 1 \\
\hline
\end{tabular}

Nota. AFA: Atitudes frente ao álcool; AFM: Atitudes frente à maconha; AFD: Atitudes frente às drogas. ${ }^{*} p<0,01$.

Como suposto, observou-se que AFA se relacionam positivamente com AFM $(r=0,60)$ e com AFD $(r=0,56)$. Foi verificado também que AFD se relacionam positivamente e mais fortemente com AFM $(r=0,76)$. Este padrão de correlações lineares pode ser visualizado graficamente na Figura 1, abaixo.

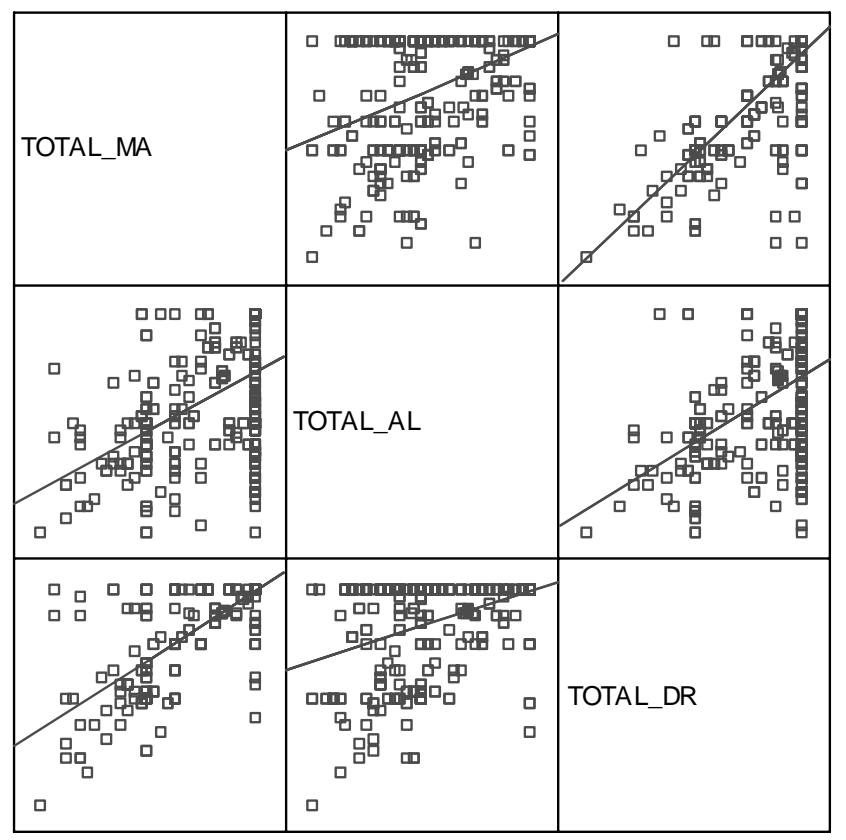

Atitudes frente ao álcool $\rightarrow$ Atitudes frente à maconha $\rightarrow$ Atitudes frente às drogas

Figura 1. Gráfico das correlações entre atitudes frente ao uso de álcool, maconha e drogas

Para apresentação dos resultados desta pesquisa, utilizou-se a nomenclatura para análises de regressão: Variável preditora $(\mathrm{X})$, mediadora $(\mathrm{M})$ e critério $(\mathrm{Y})$ : Sendo $\mathrm{X}=$ Atitudes frente ao álcool (AFA); $\mathrm{M}=$ Atitudes frente à maconha $(\mathrm{AFM})$ e $\mathrm{Y}=$ Atitudes frente às drogas (AFD). 
Estas análises verificaram a seqüência AFA $\rightarrow$ AFM $\rightarrow$ AFD.

Seguiram-se, portanto, os passos recomendados para detectar efeitos de mediação (Baron \& Kenny, 1986). O primeiro passo foi verificar se $\mathrm{X} \rightarrow \mathrm{Y}$ e o segundo passo se $\mathrm{X}$ $\rightarrow$ M. Neste sentido, realizaram-se as análises de regres- são linear; tendo, na primeira análise, como variável independente AFA para predizer AFD e, na segunda análise, esta mesma VI para predizer AFM.

Os terceiro e quarto passos consistiram em verificar se $\mathrm{X}$ e $\mathrm{M} \rightarrow \mathrm{Y}$ e se $\mathrm{X} \rightarrow \mathrm{Y}=0$ ao controlar-se $\mathrm{M}$, este procedimento consistiu na análise de regressão apresentada na Tabela 3.

Tabela 2

Análise de Regressão Linear para a Predição de AFD e AFM

\begin{tabular}{llllllll}
\hline Variáveis & $\mathrm{R}$ & $\mathrm{R}^{2}$ & $\mathrm{~F}$ & Sig $(\mathrm{F})$ & Beta & $t$ & $p$ \\
\hline X: AFA & & & & & & & \\
Y: AFD & 0,56 & 0,31 & 121,187 & 0,000 & 0,56 & 11,008 \\
M: AFM & 0,60 & 0,36 & 149,133 & 0,000 & 0,60 & 12,212 & 0,000 \\
\hline
\end{tabular}

Nota: $\mathrm{X}=$ Predictor; Y: Critério; $\mathrm{M}=$ Mediadora.

Tabela 3

Análise de Regressão Linear Múltipla para a Predição de AFD

\begin{tabular}{|c|c|c|c|c|c|c|c|c|}
\hline VI's & Beta & $t$ & $p$ & $\mathrm{CP}$ & $\mathrm{R}$ & $\mathrm{R}^{2}$ & $\mathrm{~F}$ & Sig $(F)$ \\
\hline AFA & 0,15 & 2,893 & 0,004 & 0,18 & 0,77 & 0,59 & 93,706 & 0,000 \\
\hline AFM & 0,66 & 13,274 & 0,000 & 0,63 & & & & \\
\hline Sexo & 0,02 & 0,555 & 0,579 & & & & & \\
\hline Idade & 0,03 & 0,859 & 0,391 & & & & & \\
\hline
\end{tabular}

Nota. CP = Correlação Parcial.

De acordo com esta seqüência de análises de regressão, considerando o controle da variável mediadora pela análise de correlação parcial no último passo, conclui-se que a relação entre AFA e AFD é mediada parcialmente pelas AFM, isto é: Atitudes frente ao álcool $\rightarrow$ Atitudes frente à maconha $\rightarrow$ Atitudes frente às drogas. A predição das AFD é visualizada nos gráficos da Figura 2, que mostra a linha de melhor aderência para as duas regressões simples (simple; AFA e AFM como preditoras de AFD) e o plano tridimensional para a predição de AFD conjuntamente por AFA e AFM (regressão múltipla; 3-D).

\section{Discussão}

De acordo com as análises efetuadas, pôde-se verificar inicialmente que todas as atitudes estão correlacionadas. Adicionalmente, como foco principal, verificou-se o poder preditivo destas atitudes e também sua função mediadora. Em acréscimo, testou-se ainda a influência de duas variáveis sócio-demográficas: sexo e idade. Com base nestes achados, destacaram-se as principais contribuições do presente estudo.

\section{Limitações da Pesquisa}

Como todo empreendimento científico, este também não está ausente de limitações. Neste sentido, deve-se destacar principalmente a técnica de extração da amostra, do tipo não-probabilística. Além disso, esta se constituiu exclusivamente de estudantes universitários, sendo maioria do ensino público, e de uma região, do nordeste brasileiro. Adite-se a isto o fato de não se poder contar com outras variáveis para a composição do modelo teórico. De fato, este é um modelo fundamentalmente atitudinal, exceto pelas variáveis sócio-demográficas consideradas. Deste modo, também não se acredita que seja uma panacéia para um problema cuja natureza é tão complexa. No entanto, um controle adicional poderia ser utilizado com uma variável mais social para contrastar com a variável mais pessoal, como é a atitude, considerada no contexto da teoria da ação racional (TAR).

\section{Contribuições do Presente Estudo}

Através deste estudo, pôde-se observar que: (a) AFA predizem AFD; (b) AFA predizem AFM; (c) AFA e AFM predizem AFD; (d) AFA predizem mais fortemente AFM do que AFD; (e) AFM agem como variável mediadora da relação AFA $\rightarrow$ AFD.

Não obstante, de acordo com o último passo para verificar a mediação, conclui-se a favor de um efeito de mediação parcial, isto é: a relação entre AFA e AFD é mediada parcialmente pelas AFM. Além disso, pode-se dizer que AFM moderou a relação entre AFA e AFD, no sentido de que mudou a força desta relação (Baron \& Kenny, 1986). Outra constatação deste estudo é que estas relações foram 
Pimentel, C. E., Coelho Júnior, L. L. \& Aragão, T. A. (2009). Atitudes Frente ao Uso de Álcool, Maconha e Outras Drogas: Verificando Relações de Predição e Mediação.
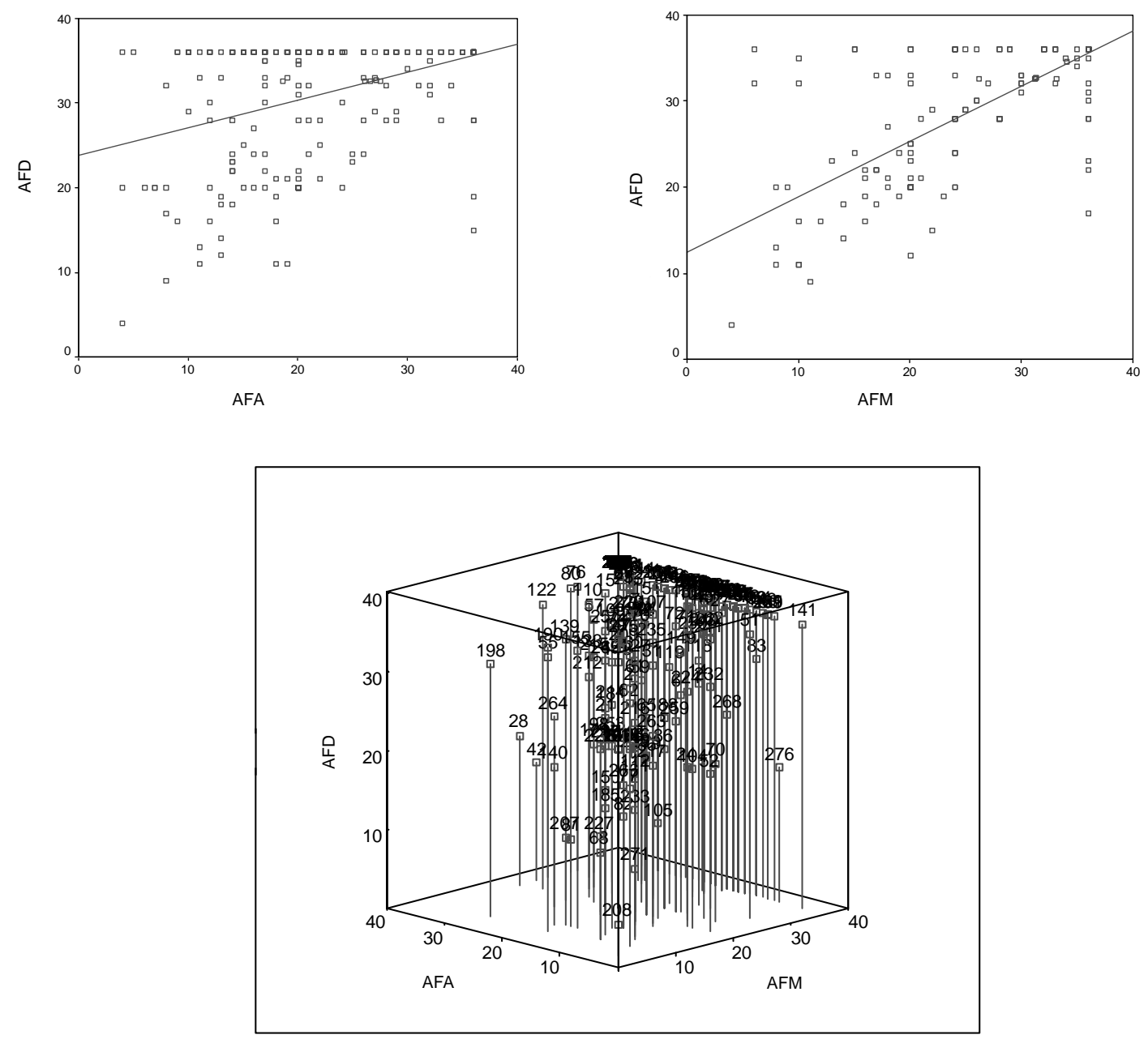

Figura 2. Gráfico da predição das atitudes frente ao uso de drogas pelas atitudes frente ao uso de álcool e maconha.

independentes das variáveis sócio-demográficas consideradas. De fato, na equação de regressão múltipla verificouse que o sexo e a idade dos participantes não se mostraram estatisticamente significativos para a predição das atitudes frente às drogas. Ressalte-se, porém, que, quando se faz referência à idade, trata-se majoritariamente de jovens. Com relação ao sexo, destaque-se que a maioria da amostra foi formada por estudantes (universitários) do sexo feminino. Estas características, portanto, devem ser tidas em consideração na interpretação destes resultados.

Embora possamos contemplar, na literatura especializada, pesquisas que mostram o desenvolvimento do uso de drogas, de drogas consideradas leves (como álcool, maconha, solventes) para drogas mais pesadas (como cocaína, heroína ou LSD), além do papel das atitudes frente ao uso de substância no comportamento de uso (Hawkins et al., 1992; Petraits et al., 1995; Simons \& Carey, 1998, 2000; Simons \& Gaher, 2004), não se encontrou nenhum estudo que verificasse as associações, considerando o construto de atitudes, frente ao uso de álcool, maconha e outras drogas. No presente estudo, considerou-se ainda a seqüência de predição destas atitudes e relações de mediação.

Em suma, verificou-se empiricamente que as atitudes frente ao uso de álcool e maconha predizem o uso de drogas, e que as atitudes frente ao uso de maconha agem como mediadoras da relação entre atitudes frente ao álcool e às drogas e como preditoras das atitudes frentes às drogas. Destaque-se, pois, este como o principal contributo da presente pesquisa que pode ser incorporado nas chamadas pathways theory of drug use. Este conhecimento pode ser útil ainda para os programas de prevenção (Blaya \& Antón, 1993) e mudança de atitudes frente ao uso destas substâncias (Scott, 1996). Uma vez que se verificou que estas atitudes estão inter-relacionadas, esta consistência cognitiva pode favorecer a mudança de atitudes (incrementando atitudes negativas ou mudando as neutras para negativas ou as positivas para negativas), mediante comunicação de massas (usando-se comunicações persuasivas em campanhas na TV ou cinema, p. ex.), em que, se intervindo em atitudes frente a determinadas substâncias, poder-se-ia, conseqüentemente, modificar outras também. Esta área 
conta com uma longa e sólida tradição de pesquisas experimentais com uso de teorias da psicologia social (Rodrigues, Assmar, \& Jablonski, 2000).

Entretanto, é importante que as relações aqui reportadas sejam verificadas em outras regiões, considerando amostras maiores e mais diversificadas, como de não-estudantes ou mesmo usuários de álcool e drogas, com o objetivo de aumentar o alcance das conclusões ora apresentadas. Dever-se-ia considerar ainda outras substâncias além das que nortearam este estudo, contribuindo para a formação de um modelo complexo de atitudes frente às drogas. Como é o caso do tabaco, que tem se demonstrado um preditor do uso de álcool e maconha (Duncan, Duncan, \& Hops, 1998).

Apesar de se verificar na literatura relações entre atitudes e comportamentos, no tocante ao consumo de substâncias, pode-se questionar que estas atitudes são de modesta e complexa predição ou mesmo que pesquisas não têm demonstrado uma conseqüente mudança de comportamentos. Porém, mesmo sendo de difícil mudança, estas atitudes são importantes para o plano de prevenção, que deve ser endereçada para todos os tipos de drogas (NIDA, 2003). Ademais, o consenso entre os pesquisadores acerca da natureza multideterminada do uso de drogas, condizente com o número de variáveis e teorias explicativas que se apresenta (Hawkins et al., 1992; Petraits et al., 1995), nos impele a ter em conta, em pesquisas futuras, variáveis de naturezas distintas, que tenham mostrado sua importância em pesquisas prévias; como os traços de personalidade e a pressão dos grupos e normas sociais (Hawkins et al., 1992; Hopwood et al., 2007; Merenäkka et al., 2003; Sussman, Dent, \& McCullar, 2000), caracterizadas como importantes peças deste "quebra-cabeças" (Petraits et al., 1995). Este amplo programa de pesquisas deve motivar ainda mais pesqui-sadores em diversas partes do nosso país, se pretende-se realmente melhor entender e lidar com um dos maiores desafios de todos os tempos, de proporções e custos mundiais (Meloni \& Laranjeira, 2004; Shedler \& Block, 1990; United Nations Office on Drugs and Crime [UNDOC], 2006).

\section{Referências}

Ajzen, I. (2001). Nature and operations of attitudes. Annual Review of Psychology, 52(1), 27-58.

Ajzen, I., \& Fishbein, M. (1980). Understanding attitudes and predicting social behavior. Englewood Cliffs, NJ: PrenticeHall.

Ajzen, I., Timko, C., \& White, J. B. (1982). Self-monitoring and the attitude-behavior Relation. Journal or Personality and Social Psychology, 42(3), 426-435.

American Psychological Association. (2002). Ethical principles of psychologists and Code of Conduct. American Psychologists, 57(12), 1060-1073.

Baron, R. M., \& Kenny, D. A. (1986). The moderator-mediator variable distinction in social psychological research: Conceptual, strategic and statistical considerations. Journal of Personality and Social Psychology, 51(6), 1173-1182.
Best, D., Rawaf, S., Rowley, J., Floyd, K., Manning, V., \& Strang, J. (2000). Drinking and smoking as concurrent predictors of illicit drug use and positive drug attitudes in adolescents. Drug and Alcohol Dependence, 60(3), 319-321.

Blaya, M. C., \& Antón, D. M. (1993). Prevención de drogodependencias: intervencion educativa versus comportamental. Anales de Psicología, 9(1), 31-42.

Blaze-Temple, D., \& Lo, S. K. (1992). Stages of drug use: A community survey of Perth teenagers. British Journal of Addiction, 87(2), 215-225.

Coelho, L. L., Jr., \& Gontiès, B. (2000). Consumo de álcool e maconha entre adolescentes: Uma perspectiva psicossocial do tema. Arquivos Brasileiros de Psicologia, 52(4), 45-58.

Colle, X., \& Curtet, F. (1983). Toxicomanie: premier produit. $L$ ' information Psychiatrique, 10(1), 1123-1132.

Correia, C. J., Simons, J., Carey, K. B., \& Borsari, B. E. (1998). Predicting drug use: Application of behavioral theories of choice. Addictive Behaviors, 23(5), 705-709.

Crites, S. L., Fabrigar, L. R., \& Petty, R. E. (1994). Measuring the affective and cognitive properties of attitudes: Conceptual and methodological issues. Personality and Social Psychology Bulletin, 20(6), 619-634.

Donnermeyer, J. F., \& Huang, T. C. (1991). Age and alcohol, marijuana and hard drug use. Journal of Drug Education, 21(3), 255-268.

Duncan, S. C., Duncan, T. E., \& Hops, H. (1998). Progressions of alcohol, cigarette, and marijuana use in adolescence. Journal of Behavioral Medicine, 21(4), 375-388.

Ellickson, P. L., Hays, R. D., \& Bell, R. M. (1992). Stepping through the drug use sequence: Longitudinal scalogram analysis of initiation and regular use. Journal of Abnormal Psychology, 101(3), 444-451.

Enriquez-Flores, I. E., \& Luis, M. A. V. (2004). Use and attitudes about drugs among nursing students at the Universidad Mayor de San Andres [Special issue]. Revista Latino-Americana de Enfermagem, 12, 376-382.

Fishbein, M., \& Ajzen, I. (1975). Belief, attitude, intention, and behavior: An introduction to theory and research. Reading, MA: Addison-Wesley.

Golub, A., \& Johnson, B. D. (1994). The shifting importance of alcohol and marijuana as gateway substances among serious drug abusers. Journal of Studies on Alcohol, 55(5), 607-614.

Gouveia, V. V., Pimentel, C. E., Medeiros, E. D., Gouveia, R. S., \& Palmeira, J. N. (2007). Escala de atitudes frente ao uso de drogas: Evidências de validade fatorial e preditiva. Jornal Brasileiro de Psiquiatria, 56(1), 53-59.

Gouveia, V. V., Pimentel, C. E., Queiroga, F., Meira, M., \& Jesus, G. R. (2005). Escala de atitudes frente ao uso de maconha: Comprovação da sua validade de construto. Jornal Brasileiro de Psiquiatria, 54(1), 5-12.

Hawkins, J. D., Catalano, R. F., \& Miller, J. Y. (1992). Risk and protective factors for alcohol and other drug problems in adolescence and early adulthood: Implications for substance abuse prevention. Psychological Bulletin, 112(1), 64-105.

Holland, R. W., Verplanken, B., \& van Knippenberg, A. (2002). On the nature of attitude-behavior relations: The strong guide, the weak follow. European Journal of Social Psychology, 32, 869-872.

Hopwood, C. J., Morey, L. C., Skodol, A. E., Stout, R. L., Yen, S., Ansell, E. B., Grilo, C. M., \& McGlashan, T. H. (2007). Five-factor model personality traits associated with alcoholrelated diagnoses in a clinical sample. Journal of Studies on Alcohol and Drugs, 68(3), 455-460. 
Pimentel, C. E., Coelho Júnior, L. L. \& Aragão, T. A. (2009). Atitudes Frente ao Uso de Álcool, Maconha e Outras Drogas: Verificando Relações de Predição e Mediação.

Isacson, D., \& Bingefors, K. (2002). Attitudes towards drugs: A survey in general population. Pharmacy World \& Science, 24(3), 104-110.

Kerr-Corrêa, F., Andrade, A. G., Bassit, A. Z., \& Boccuto, N. M. V. F. (1999). Uso de álcool e drogas por estudantes de Medicina da Unesp. Revista Brasileira de Psiquiatra, 21, 95-100.

Kim, M. S., \& Hunter, J. E. (1993). Attitude-behavior relations: A meta-analysis of attitudinal relevance and topic. Journal of Communication, 43(1), 101-142.

Meloni, J. N., \& Laranjeira, R. (2004). Custo social e de saúde do consumo de álcool. Revista Brasileira de Psiquiatria, 26(1), 7-10.

Merenäkka, L., Harroa, M., Kiiveb, E., Laidrab, K., Eensooa, D., Allikb, J., et al. (2003). Association between substance use, personality traits, and platelet MAO activity in preadolescents and adolescents. Addictive Behaviors, 28, 1507 1514.

National Institute on Drug Abuse. (2003). Preventing drug use among children and adolescents: A research-based guide for parents, educators and community leaders. New York: Author.

Noto, A. R., Baptista, M. C., Faria, S. T., Nappo, S., Galduróz, J. F. C., \& Carlini, E. A. (2003). Drugs and health in the Brazilian press: An analysis of articles published in newspapers and magazines. Cadernos de Saúde Pública (Rio de Janeiro), 19(1), 69-79.

Petraits, J., Flay, B. R., \& Miller T. Q. (1995). Reviewing theories of adolescent substance use: Organizing pieces in the puzzle. Psychological Bulletin, 117(1), 67-86.

Roazzi, A. (1987). Considerações sobre o significado ideológico das toxicomanias. Arquivos Brasileiros de Psicologia, 4, 4864.

Rodrigues, A., Assmar, E. M. L., \& Jablonski, B. (2000). Psicologia social. Petrópolis, RJ: Vozes.

Sanchez, Z. van der M., \& Nappo, S. A. (2002). Progression on drug use and its intervening factors among crack users. Revista de Saúde Pública (São Paulo), 36(4), 420-430.

Scott, C. G. (1996). Understanding attitude change in developing effective substance abuse prevention programs for adolescents. School Counselor, 43(3), 187-195.

Shedler, J., \& Block, J. (1990). Adolescent drug use and psychological health. A longitudinal inquiry. American Psychologist, 45(5), 612-630.

Simons, J., \& Carey, K. B. (1998). A structural analysis of attitudes toward alcohol and marijuana use. Personality and Social Psychology Bulletin, 24(7), 727-735.

Simons, J., \& Carey, K. B. (2000). Attitudes toward marijuana use and drug-free experience: Relationships with behavior. Addictive Behaviors, 25(3), 323-331.

Simons, J. S., \& Gaher, R. M. (2004). Attitudes toward alcohol and drug-free experience among college students: Relationships with alcohol consumption and problems. American Journal of Drug and Alcohol Abuse, 31(2), 337-356.

Smith, J. R., \& Terry, D. J. (2003). Attitude-behaviour consistency: The role of group norms, attitude accessibility, and mode of behavioural decision-making. European Journal of Social Psychology, 33(5), 591-608.

Sussman, S., Dent, C. W., \& McCullar, W. J. (2000). Group selfidentification as a prospective preditor of drug use and violence in high-risk youth. Psychology of Addictive Behavior, 14(2), 192-196.

United Nations Office on Drugs and Crime. (2006). World Drug Report (Vol. 1). New York: Author.

Wicker, A. W. (1969). Attitudes versus actions: The relationship of verbal and overt behavioral responses to attitude objects. Journal of Social Issues, 25(4), 41-78.

Recebido: 10/07/2007 